\title{
Pre versus Post-Operative Initiation of Warfarin Therapy in Patients undergoing Total Hip and Knee Arthroplasty
}

\section{Cara Cipriano ${ }^{1}$, Nicholas Erdle ${ }^{2}$, Kai $\mathrm{Li}^{3}$ and Brian Curtin ${ }^{4 *}$}

${ }^{1}$ Washington University in St Louis, St Louis, MO, USA

${ }^{2}$ Resident, Naval Medical Center, Portsmouth, ME, USA

${ }^{3}$ Resident, Virginia Commonwealth University, Richmond, VA, USA

${ }^{4}$ OrthoCarolina, Charlotte, NC, USA

\begin{abstract}
Background: The optimal strategy for postoperative Deep Venous Thrombosis (DVT) prophylaxis remains among the most controversial topics in hip and knee arthroplasty. Warfarin, the most commonly used chemical anticoagulant, initially causes transient hypercoagulability; however the optimal timing of treatment with respect to surgery remains unclear. Our purpose was to evaluate the effects of pre- versus postoperative initiation of warfarin therapy with a primary endpoint of perioperative change in hemoglobin (pre- minus post-operative level), with secondary endpoints of postoperative International Normalized Ratio (INR), drain output, and bleeding/thrombotic events.

Methods: A quasi-experimental study design was employed, under which patients were assigned to begin taking warfarin the night prior to surgery or the night following surgery based on day of the week seen in clinic. A prior power analysis was conducted in order to ensure appropriate enrollment to detect a $0.5 \mathrm{~g} / \mathrm{dL}$ difference in perioperative change in hemoglobin between groups, given an alpha level of 0.05 and beta of 0.80 . Based on the results, the study included all primary, elective total hip and knee arthroplasties performed by a single surgeon over a 12 month period. Fifteen patients were excluded (7 chronic anticoagulation, 3 hip fractures, 2 medical contraindications, 3 simultaneous procedures), leaving 165 cases (108 hips, 57 knees) available for study. Of these, 73 received warfarin preoperatively (49 hips, 24 knees) and 92 postoperatively (59 hips, 33 knees). Warfarin was dosed according to a standard nomogram in both groups. INR (on postoperative days 1 and 2), perioperative decrease in hemoglobin (difference between level preoperatively and on postoperative days 1 and 2), and drain outputs were compared between groups using a student $\mathrm{t}$ test. Adverse events (transfusions, hematomas, epidural complications, and pulmonary embolus) were compared using two-tailed Fischer's exact test.
\end{abstract}

Results: No statistically significant difference in perioperative hemoglobin change was observed between groups on either postoperative day 1 (mean 3.279 versus $3.377, p=0.6824$ ) or 2 (mean 4.0 versus $4.12, p=0.6831$ ). As expected, the preoperative warfarin group demonstrated higher INRs on both postoperative days 1 (mean 1.18 versus $1.12, p=0.0023$ ) and 2 (mean 1.46 versus $1.31, p=0.0006$ ). Of note, the preoperative warfarin group also demonstrated significantly lower drain outputs (mean 185.4 versus $268.7, p=0.0025$ ). 9 transfusions (4 preoperative dosing, 5 postoperative dosing), 3 hematomas (1 preoperative dosing, 2 postoperative dosing), and 1 pulmonary embolus (preoperative dosing) occurred, but no significant difference could be detected given the numbers available for study.

Conclusions: Initiation of warfarin pre- rather than postoperatively was not associated with a significant difference in perioperative hemoglobin change, although a significant reduction in drain output was observed. Larger studies are needed to determine whether the risk of adverse events is increased with either strategy.

Keywords: Hip arthroplasty; Knee arthroplasty; Vitamin K; Hemoglobin; Spinal anesthesia; Prophylaxis; Thrombotic; Bleeding; Anticoagulation

\section{Introduction}

The optimal strategy for postoperative deep venous thrombosis (DVT) prophylaxis remains a controversial topic in hip and knee arthroplasty. While it has been widely accepted that some form is required, a consensus on the ideal modality has not been established [1]. The benefits of chemical DVT prophylaxis must be balanced against the risks of anticoagulation in the early post-operative period, as increased bleeding can necessitate transfusions as well as lead to hematomas and other wound healing complications.

Warfarin therapy is the most commonly employed form of chemical DVT prophylaxis following hip and knee arthroplasty in the United States [2]. It is usually administered beginning the evening after surgery and titrated according to International Normalized Ratio (INR) with a target range of 1.6-3.0, depending on the institution and surgeon [2]. It acts by preventing the carboxylation of Vitamin $\mathrm{K}$ dependent clotting factors in the liver; however, it first affects anti-coagulant Protein $\mathrm{C}$ and $\mathrm{S}$, leading to an interval of transient hypercoagulability. While the risk of DVT formation may begin at the time of surgery or during the early postoperative period, patients are unprotected until their INRs reach

${ }^{*}$ Corresponding author: Brian Curtin, 2001 Vail Avenue, Suite 200A, Charlotte, NC 28207, MD, USA, Tel: 704.323.2564; E-mail: bmcurt01@gmail.com

Received May 05, 2015; Accepted June 02, 2015; Published June 15, 2015

Citation: Cipriano C, Erdle N, Li K, Curtin B (2015) Pre versus Post-Operative Initiation of Warfarin Therapy in Patients undergoing Total Hip and Knee Arthroplasty. J Arthritis 4: 156. doi:10.4172/2167-7921.1000156

Copyright: (c) 2015 Cipriano C, et al. This is an open-access article distributed under the terms of the Creative Commons Attribution License, which permits unrestricted use, distribution, and reproduction in any medium, provided the original author and source are credited. 
appropriate levels [3]; thus, the optimal timing of warfarin treatment with respect to surgery remains unclear.

Our purpose was to evaluate the effects of pre versus postoperative initiation of warfarin therapy on postoperative International Normalized Ratio (INR), perioperative blood loss, and related complications.

\section{Patients and Methods}

This quasi-randomized controlled study included all primary, elective total hip and knee arthroplasties (THA, TKA) performed by the senior author $(\mathrm{BC})$ at a single institution over a 12 month period (January 2012 - January 2013). Patients were assigned to begin taking warfarin the night prior to surgery or the night following surgery based on day of the week evaluated in clinic; those seen on Mondays and Wednesdays were prescribed $5 \mathrm{mg}$ warfarin the evening before surgery, while those seen on Friday began warfarin on the evening following surgery. An a priori power analysis was performed to ensure appropriate sample size to detect a $0.5 \mathrm{~g} / \mathrm{dL}$ difference in perioperative change in hemoglobin between groups, given an alpha level of 0.05 and beta of 0.80 . The results indicated that 64 patients would be required in each group, or at least 140 total when allowing for an estimated $10 \%$ exclusion rate.

Demographic distribution of patients assigned to each group is shown in Table 1. Preoperative hemoglobin levels were measured on all patients within 2 weeks of surgery. Duramorph spinal anesthesia was routinely employed, and all TKAs were performed using a tourniquet, which was inflated at the time of incision and deflated prior to closure. A single medium HemoVac drain ( 10 French $/ 0.125 \mathrm{in} / 0.32 \mathrm{~cm}$ diameter) was placed at the end of each case and discontinued on the morning of postoperative day (POD) 1 . All patients received $5 \mathrm{mg}$ of warfarin at 10 $\mathrm{pm}$ on the evening following surgery (6-12 hours postoperatively), and a standard nomogram was used to titrate warfarin dosing according to INR levels in both patient groups thereafter. The surgeon and other staff were blinded to the patients' anticoagulation protocols at the time of surgery and throughout their hospitalizations.

Following appropriate Institutional Review Board approval, the electronic medical records for patients in the study population were retrospectively reviewed for INR levels (on POD 1 and 2), drain outputs (on POD 1, when all drains were removed), and change between pre and postoperative hemoglobin levels (on POD 1 and 2). Patients were monitored clinically, but no Doppler studies or other screening modalities were performed to detect asymptomatic DVTs. The number of adverse events related to anticoagulation (wound healing complications, hematomas, epidural complications, and transfusions) or thrombosis (symptomatic DVT, pulmonary embolus) was also noted. These outcomes were compared between patient populations using a chi-square test for categorical variables (wound healing complications, hematomas, and transfusions) and student t-test for continuous variables (postoperative INR, drain output, and change between pre- and postoperative hemoglobin levels). Adverse events (transfusions, hematomas, epidural complications, symptomatic DVT, and pulmonary embolus) were compared using two-tailed Fischer's exact test.

\section{Results}

Of the 177 patients initially reviewed, 12 were excluded: 7 receiving chronic anticoagulation for treatment of another condition, 3 undergoing simultaneous procedures that would likely increase blood loss $(2$ significant hardware removals and 1 contralateral core decompression), and 2 with medical contraindications to warfarin (1 hemophiliac, 1 other intolerance). Of the remaining 165 cases (108 THA, 57 TKA) available for study, 73 were prescribed warfarin preoperatively (49 THA, 24 TKA) and 92 postoperatively (59 THA, 33 TKA). Patients were evenly distributed between groups in terms of gender and hip versus knee arthroplasty $(p=0.3429$ and 0.7431 , respectively), although those who received postoperative warfarin were slightly older (mean 59.6 compared to 54.4 years, $\mathrm{p}=0.0034$ ). Five patients from the study group and 2 patients from the control group were discharged on POD 1 and therefore excluded from the analysis of INR and hemoglobin on POD 2 (Table 1). In addition, drain outputs were not reliably documented in 9 patients from the preoperative treatment group ( 5 not recorded, 1 fell out) and 6 patients from the postoperative treatment group (6 not recorded, 3 fell out), so these patients were excluded from the analysis of drain output.

No significant difference in perioperative change in hemoglobin was observed between groups on either POD1 (mean 3.279 versus $3.377, \mathrm{p}=0.6824$ ) or POD2 (mean 4.0 versus $4.12, \mathrm{p}=0.6831$ ). The study group demonstrated higher INRs on POD1 (mean 1.18 versus 1.12 , $\mathrm{p}=0.0023$ ) and POD2 (mean 1.46 versus $1.31, \mathrm{p}=0.0006$ ), with more patients achieving therapeutic INR $(\geq 1.8)$ by POD2 (7.9\% compared to $3.4 \%)$. This group also found to have statistically significantly lower drain outputs (mean 185.4 versus $268.7, \mathrm{p}=0.0025$ ). Nine transfusions (4 study patients, 5 control patients), 3 hematomas ( 1 study patients, 2 control patients), 1 pulmonary embolus (study patient), 0 other symptomatic DVTs, and 0 epidural-related complications occurred; no significant difference in the rate of these events could be detected given the numbers available for study (Table 2).

\section{Discussion}

The critical importance of DVT prophylaxis following THA and TKA has been well established; however, the optimal agent and time of initiation to minimize both thrombotic and bleeding events remain widely debated. The ideal form of prophylaxis would provide anticoagulation during the period of greatest risk for thrombosis without increasing rates of wound healing complications, hematomas, and acute blood loss anemia necessitating transfusion.

\begin{tabular}{|c|c|c|c|}
\hline & Study Group & Control Group & p value \\
\hline THA $(n)$ & 49 & 59 & \multirow{2}{*}{0.7431} \\
\hline TKA (n) & 24 & 33 & \multirow{2}{*}{0.3429} \\
\hline Male (n) & 29 & 45 & 0.0034 \\
\hline Female (n) & 43 & 47 & 0.4 \\
\hline Age (mean) & 59.6 & 54.4 & 0.000 \\
\hline
\end{tabular}

Table 1: Demographic distribution of patients assigned to pre compared to postoperative initiation of warfarin treatment.

\begin{tabular}{|c|c|c|c|}
\hline & Study Group & Control Group & p value \\
\hline INR (mean, POD 1) & 1.18 & 1.12 & 0.0023 \\
\hline INR (mean, POD 2) & 1.46 & 1.31 & 0.0006 \\
\hline Drain output (mean) & 185.4 & 268.7 & 0.0025 \\
\hline$\Delta \mathrm{Hg}$ (mean, POD 1) & 3.28 & 3.38 & 0.6824 \\
\hline$\Delta \mathrm{Hg}$ (mean, POD 2) & 4.00 & 4.12 & 0.6831 \\
\hline Transfusions (n) & 4 & 5 & 1.000 \\
\hline Hematomas (n) & 1 & 2 & 1.000 \\
\hline Pulmonary emboli (n) & 1 & 0 & 0.4398 \\
\hline Symptomatic DVTs (n) & 0 & 0 & $\mathrm{n} / \mathrm{a}$ \\
\hline Epidural complications $(\mathrm{n})$ & 0 & 0 & $\mathrm{n} / \mathrm{a}$ \\
\hline
\end{tabular}

Table 2: Effects of pre- compared to postoperative initiation of warfarin therapy on patient INR, drain output, and perioperative drop in hemoglobin, as well as pertinent complication rates. 
While thrombi theoretically begin to form intraoperatively with the combination of venous stasis and surgical trauma, the majority seems to develop in the early postoperative period and may continue to evolve over the ensuing weeks or months [4,5], potentially leading to readmission or delayed complications [6,7]. In order to address this concern, the optimal timing of therapy using low molecular weight heparin (LMWH) and other relatively new anticoagulants for THA and TKA has been extensively researched [3,8-14]. The aggregate of this literature indicates that preoperative initiation is not necessary for effective prophylaxis, that initiation between 2 hours pre and 6 hours postoperatively increases the risk of major bleeds, and that initiation at 6 hours post operatively is both safe and likely more efficacious than delayed administration at 12-24 hours [11,12]. This suggests that anticoagulation is less beneficial, or even potentially harmful, during and immediately following surgery itself, but extended delays may be less effective in preventing thrombus development.

The issue of anticoagulation timing has not been addressed with respect to warfarin, in spite of the fact that it is the most common form of DVT prophylaxis employed by members of the American Association of Hip and Knee Surgeons [2]. Warfarin therapy is typically initiated on the night following surgery; however, its mechanism of action is known to cause transient hypercoagulability due to suppression of proteins $\mathrm{C}$ and $\mathrm{S}$ that precedes suppression of Vitamin K-dependent clotting factors $[15,16]$. According to the literature described above, this initial hypercoagulability may be more advantageous during the period of greatest blood loss (i.e., intra- and immediately postoperatively) than during the days following surgery, when the patient might be at risk for thrombus development and propagation.

Our study did not identify a statistically significant advantage of one warfarin-dosing strategy over the other, although a significant decrease in drain output was observed in the group that received preoperative warfarin $(p=0.0025)$. Mean output for the preoperative treatment group was $185.4 \mathrm{~mL}$ compared to $268.7 \mathrm{~mL}$ for the postoperative treatment group, resulting in an average difference of $83.3 \mathrm{~mL}$ that we consider clinically relevant. We also observed statistically significantly higher INRs in the study population on both POD 1 and 2 ( $\mathrm{p}=0.0023$ and 0.0006 , respectively); while the difference may not be clinically significant at that time point, it does suggest that the study group would reach therapeutic INR levels for DVT prophylaxis one day sooner, as expected. Given the numbers available for study, no significant difference in the rates of thrombotic or bleeding complications could be detected.

This study has several limitations that must be acknowledged. Patients were not strictly randomized, but assigned to their treatment groups based on clinic day of the week in a quasi-experimental observational study design. Mean age was slightly younger in the study group (54.4 versus 59.6 years, $\mathrm{p}=0.0034$ ); however, this difference would not likely impact our results, and the patient distribution was otherwise even with respect to gender and hip versus knee arthroplasty ( $\mathrm{p}=0.3429$ and 0.7431 , respectively). Nine (12.3\%) patients in the study group and $6(6.5 \%)$ in the control groups were excluded from analysis of drain output, primarily due to insufficient documentation, and this data was considered missing completely at random. Most importantly, perhaps, a larger sample population would be needed to compare these warfarin dosing strategies in terms of rare but important complications related to bleeding or thrombosis.

In addition to these study-specific limitations, the evaluation of perioperative bleeding is subject to several inherent challenges. Minor discrepancies in surgical technique, patient size, or difficulty of the case can contribute to rates of intra or postoperative blood loss. Serum hemoglobin may be affected by the hydration status of the patient, which in turn depends on several variables, such as volume of intravenous fluids administered. Multiple risk factors may contribute to wound healing complications, and the decision to transfuse is made on an individual case basis according to symptoms and other patientspecific factors. In this study, data on intraoperative estimated blood loss was not collected due to the potential for imprecise measurement and subjective bias; instead, drain output was selected as a more reliable measure of the patient's propensity to bleed in the perioperative period. Given that drain output averaged $83.3 \mathrm{~mL}$ per case lower following preoperative warfarin, a similar reduction in blood loss may have occurred during surgery itself but would not have been detected in this study.

Finally, patient morbidity and mortality due to pulmonary embolus, which remain the most clinically relevant outcomes, occur so infrequently that they can only be feasibly evaluated in large database studies or meta-analyses. Our study, like the vast majority of the literature on this subject, was not powered to compare the rates of symptomatic DVT, nor was this our goal. Rather, it was intended to evaluate INR, drain output, and change in hemoglobin; therefore, as in most publications, the sample size is too small to determine significant differences in the rates of major adverse events.

\section{Conclusion}

In our study of 165 patients undergoing primary, elective THA or TKA, initiation of warfarin therapy on the night prior to surgery compared to the night following surgery was associated with significantly decreased drain output and earlier increases in postoperative INR; however, we did not observe a statistically significant difference in perioperative change in hemoglobin. While we were unable to detect any difference in complication rates between groups, larger studies are needed to more definitively determine whether the risk of adverse events is decreased with either strategy.

\section{References}

1. http://www.aaos.org/research/guidelines/VTE/VTE_full_guideline.pdf

2. Markel DC, York S, Liston MJ Jr, Flynn JC, Barnes CL, et al. (2010) Venous Thromboembolism: management by American Association of Hip and Knee Surgeons. J Arthroplasty 25: 3-9.

3. Warwick D, Rosencher N (2010) The "critical thrombosis period" in major orthopedic surgery: when to start and when to stop prophylaxis. Clin App Thromb Hemost 16: 394-405.

4. Arcelus JI, Monreal M, Caprini JA, Guisado JG, Soto MJ, et al. (2008) Clinica presentation and time-course of postoperative venous thromboembolism: Results from the RIETE Registry. Thromb Haemost 99: 546-551.

5. Bjørnarå BT, Gudmundsen TE, Dahl OE (2006) Frequency and timing of clinical venous thromboembolism after major joint surgery. J Bone Joint Surg $\mathrm{Br}$ 88: 386-391.

6. Seagroatt V, Tan HS, Goldacre M, Bulstrode C, Nugent I, et al. (1991) Elective total hip replacement: incidence, emergency readmission rate, and postoperative mortality. BMJ 303: 1431-1435.

7. White RH, Romano PS, Zhou H, Rodrigo J, Bargar W (1998) Incidence and time course of thromboembolic outcomes following total hip or knee arthroplasty. Arch Intern Med 158: 1525-1531.

8. Hull RD, Brant RF, Pineo GF, Stein PD, Raskob GE, et al. (1999) Preoperative vs postoperative initiation of low-molecular-weight heparin prophylaxis against venous thromboembolism in patients undergoing elective hip replacement. Arch Intern Med 159: 137-141.

9. Hull RD, Pineo GF, Stein PD, Mah AF, Maclsaac SM, et al. (2001) Timing of initial administration of low-molecular-weight heparin prophylaxis against deep 
Citation: Cipriano C, Erdle N, Li K, Curtin B (2015) Pre versus Post-Operative Initiation of Warfarin Therapy in Patients undergoing Total Hip and Knee Arthroplasty. J Arthritis 4: 156. doi:10.4172/2167-7921.1000156

Page 4 of 4

vein thrombosis in patients following elective hip arthroplasty: a systematic review. Arch Intern Med 161: 1952-1960.

10. Kwong LM, Muntz JE (2002) Thromboprophylaxis dosing: the relationship between timing of first administration, efficacy, and safety. Am J Orthop (Belle Mead NJ) 31: 16-20.

11. Perka C (2011) Preoperative versus postoperative initiation of thromboprophylaxis following major orthopedic surgery: safety and efficacy of postoperative administration supported by recent trials of new oral anticoagulants. Thromb J 9: 17.

12. Raskob GE, Hirsh J (2003) Controversies in timing of the first dose of anticoagulant prophylaxis against venous thromboembolism after major orthopedic surgery. Chest 124: 379S-385S.
13. Strebel N, Prins M, Agnelli G, Büller HR (2002) Preoperative or postoperative start of prophylaxis for venous thromboembolism with low-molecular-weight heparin in elective hip surgery? Arch Intern Med 162: 1451-1456

14. Tribout B, Colin-Mercier F (2007) New versus established drugs in venous thromboprophylaxis: efficacy and safety considerations related to timing of administration. Am J Cardiovasc Drugs 7: 1-15.

15. Esmon CT, Vigano-D’Angelo S, D’Angelo A, Comp PC (1987) Anticoagulation proteins $C$ and S. Adv Exp Med Biol 214: 47-54.

16. Vigano D'Angelo S, Comp PC, Esmon CT, D'Angelo A (1986) Relationship between protein $C$ antigen and anticoagulant activity during oral anticoagulation and in selected disease states. J Clin Invest 77: 416-425. 